DOI: $10.1002 / c p h c .200700370$

\section{Conformational Adaptation in Supramolecular Assembly on Surfaces}

Florian Klappenberger, ${ }^{*[a, b]}$ Marta Esther CañasVentura, ${ }^{[a,}$ d] Sylvain Clair, ${ }^{[a]}$ Stéphane Pons, ${ }^{[a]}$ Uta Schlickum, ${ }^{[a]}$ Zhi-Rong Qu, ${ }^{[\mathrm{c}]}$ Harald Brune, ${ }^{[\mathrm{a}]}$ Klaus Kern, ${ }^{[a, e]}$ Thomas Strunskus, ${ }^{[f]}$ Christof Wöll, ${ }^{[f]}$ Alessio Comisso, ${ }^{\left[{ }^{[g]}\right.}$ Alessandro De Vita, ${ }^{[g]}$ Mario Ruben, ${ }^{*[c]}$ and Johannes V. Barth ${ }^{*[a, b, h]}$

Supramolecular chemistry provides an original approach to nanoscience and nanotechnology by its intrinsically molecularlevel control of self-assembly processes. ${ }^{[1]}$ The spontaneous but controlled generation of complex supramolecular entities from suitably designed molecular components opens the way to the efficient-because extremely parallel—bottom-up construction of supramolecular architectures, with specific func-

[a] Dr. F. Klappenberger, M. E. Cañas-Ventura, Dr. S. Clair, Dr. S. Pons, Dr. U. Schlickum, Prof. H. Brune, Prof. K. Kern, Prof. J. V. Barth Institut de Physique des Nanostructures

Ecole Polytechnique Fédérale de Lausanne

CH-1015 Lausanne (Switzerland)

Fax:(+49) 8928912339

E-mail: florian.klappenberger@ph.tum.de

[b] Dr. F. Klappenberger, Prof. J. V. Barth

Physik Department E20

TU München, Munich (Germany)

Fax: (+ 49) 8928912339

E-mail:jvb@ph.tum.de

[c] Dr. Z.-R. Qu, Dr. M. Ruben

Institute of Nanotechnology

Forschungszentrum Karlsruhe

Karlsruhe (Germany)

Fax: (+ 49) 7247826434

E-mail:Mario.Ruben@int.fzk.de

[d] M. E. Cañas-Ventura

Swiss Federal Laboratories for Materials Testing and Research (EMPA) Thun (Switzerland)

[e] Prof. K. Kern

Max-Planck-Institut für Festkörperforschung

Stuttgart (Germany)

[f] Dr. T. Strunskus, Prof. C. Wöll

Lehrstuhl für Physikalische Chemie I

Ruhr-Universität Bochum

Bochum (Germany)

[g] Dr. A. Comisso, Prof. A. De Vita

King's College London

Strand, London WC2R 2 LS (UK)

and

DEMOCRITOS AND DMRN-CENMAT

Università di Trieste

Trieste (Italy)

[h] Prof. J. V. Barth

PHAS-AMPEL, UBC Vancouver

Vancouver, V6T1Z4 (Canada)

Supporting information for this article is available on the WWW under http://www.chemphyschem.org or from the author. tionalities emerging at each hierarchic step of complexity. Among the underlying noncovalent interactions, hydrogen bonding has been shown to be particularly useful for structural control. ${ }^{[2]}$

For two-dimensional (2D) supramolecular engineering, the self-assembly processes are conducted at surfaces. ${ }^{[3]}$ The primary advantages of this approach are the unprecedented direct access to structural information at the atomic level by employing scanning probe techniques, notably scanning tunneling microscopy (STM), and the possibility to realize specific low-dimensional molecular structures that can eventually be integrated into nanostructured environments.

In most systems investigated to date, the transcription to $2 \mathrm{D}$ of hydrogen-bonding motifs identified in 3D systems was achieved by employing planar molecular building blocks, (socalled tectons). These adsorb in a flat geometry on surfaces, thus favoring lateral linkages between functional endgroups. ${ }^{[4]}$ In this case, the necessary balance between adsorbate-substrate and lateral intermolecular interactions can be tuned by appropriately selecting the symmetry and chemical reactivity of the substrate. It is, however, much harder to control tectons exhibiting conformational flexibility, although such species qualify for the self-assembly of particularly elaborate nanostructures. ${ }^{[5]}$ Moreover, the conformational design of single molecules and molecular layers is a crucial ingredient to achieve a targeted set of chemical and physical properties, for example, the realization of single-molecule switches, ${ }^{[6]}$ the control of chiral recognition, ${ }^{[7]}$ or efficient heterogenous catalysis. ${ }^{[8]}$ To obtain complete control over the assembly behavior of complex molecular species at surfaces, it is thus mandatory to understand the interplay of conformation and supramolecular organization.

Herein, we present a study of the surface-confined self-assembly of a conformationally flexible tecton. Specifically, we employed the oxalic amide 1 that has been designed to form structures consisting of a multitude of units that are tethered through hydrogen bonds between the amide moieties of adjacent molecules. The $N_{,} N^{\prime}$-diphenyl oxalic amide test molecule (Scheme 1) consists of two amide groups fused by a $\sigma \mathrm{C}-\mathrm{C}$ bond unit $(-\mathrm{NH}-\mathrm{C}(=\mathrm{O})-\mathrm{C}(=\mathrm{O})-\mathrm{HN}-)$. By substituting one phenyl group at each of the nitrogen atoms, lateral hydrogen bonding along the direction of the molecular backbone is blocked. In a planar projection, the molecule can adopt two different conformations with respect to the central $\sigma \mathrm{C}-\mathrm{C}$ bond: s-trans and s-cis. These can be interconverted by rotation around the central $\sigma \mathrm{C}-\mathrm{C}$ bond. However, this process is expected to be hindered on a surface by an increased energy

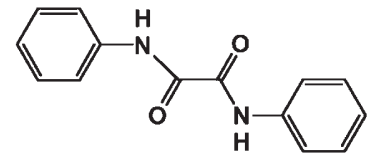

s-trans

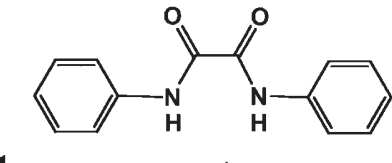

s-cis
Scheme 1. Molecular structure of $N, N^{\prime}$-diphenyl oxalic amide 1 with the two possible $2 \mathrm{D}$-confined conformations reflecting rotation about the central single $\mathrm{C}-\mathrm{C}$ bond. 
barrier owing to molecule-surface interactions, thus leading to resolution in two enantiomers for the $2 \mathrm{D}$ prochiral s-trans configuration. ${ }^{[9]}$ When the restriction of the planar confinement is lifted, the $\sigma$ bonds of the molecular backbone provide conformational flexibility. For example, one needs to consider the rotation of the phenyl moieties around the $\sigma \mathrm{N}-\mathrm{C}$ bonds, that is, the axes connecting phenyl to nitrogen.

We investigated the supramolecular organization of 1 on the reconstructed $\mathrm{Au}(111)$ surface under ultra-high vacuum (UHV) by STM and near-edge $\mathrm{X}$-ray absorption fine structure spectroscopy (NEXAFS). Our results were rationalized by modeling based on density functional theory (DFT). We find that during the assembly process from isolated molecules or small clusters into one-dimensional (1D) chains, the molecule 1 undergoes a marked conformational transformation. To optimize hydrogen bonding necessary for the chain formation, the molecules adapt their conformation to overcome otherwise interfering steric hindrance.

The molecules of $\mathbf{1}$ were deposited on the reconstructed $\mathrm{Au}(111)$ surface by sublimation under UHV conditions. Figure 1 shows a series of STM images for molecules deposited at dif-
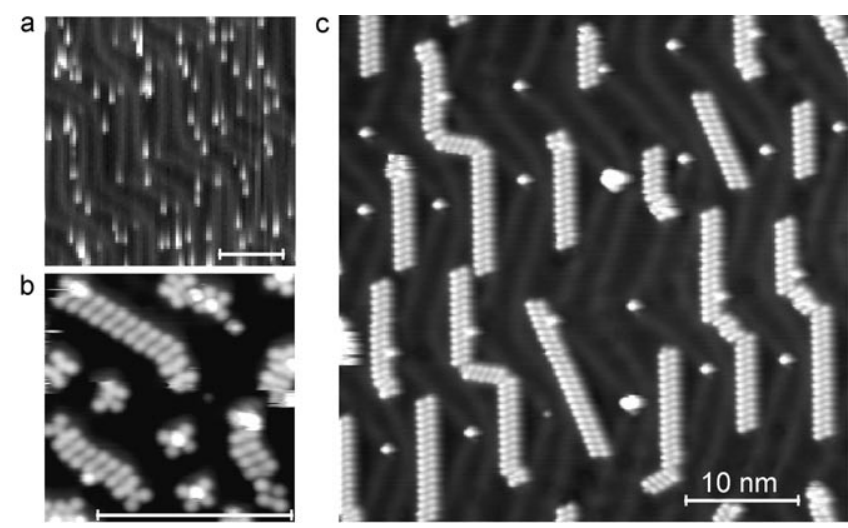

Figure 1. STM images at different temperatures. The scale bar denotes $10 \mathrm{~nm}$ in each of the three cases. a) Adsorption at low temperature $\left(T=155 \mathrm{~K}\right.$ ) yields isolated molecules. (tunneling voltage $V_{t}=-640 \mathrm{mV}$, tunneling current $I=0.28 \mathrm{nA}$ ). b) Metastable structures are found at $T=265 \mathrm{~K}$. $\left(V_{t}=-50 \mathrm{mV}, I=0.12 \mathrm{nA}\right)$. c) A high-temperature deposition $(T=300 \mathrm{~K})$ results in the self-assembly of $1 \mathrm{D}$ chains due to $\mathrm{H}$-bonds $\left(V_{t}=-200 \mathrm{mV}\right.$, $I=0.35 \mathrm{nA}$ ).

ferent sample temperatures $T$. In the pictures the zig-zag shaped, gray double lines reflect the substrate reconstruction pattern. ${ }^{[10]}$ Molecules appear as bright spots with an apparent height between 1 and $1.5 \AA$, a typical height for molecules with $\pi$-conjugated systems. Adsorption at $T=155 \mathrm{~K}$ (Figure $1 \mathrm{a}$ ) leads to separated small features, whose spatial distribution shows no significant ordering except the preferred appearance in the face-centered cubic (fcc) stacking regions. Under these conditions, it proved difficult to obtain intramolecular resolution, as the features are easily pushed aside by the STM tip. The size of the numerous, smaller spots is principally consistent with the assumption that each spot corresponds to an individual molecule, or possibly a dimer. Larger spots indicating clusters of several tectons were rarely detected. The fact that structure formation is suppressed hints at a diffusion length after adsorption that is smaller than the average intermolecular distance, which in this case is about $5 \mathrm{~nm}$.

By increasing the deposition temperature to $T=265 \mathrm{~K}$, onedimensional, chainlike assemblies of 1 evolve (Figure $1 \mathrm{~b}$ ). This chain-forming is associated with the development of hydrogen bonds between the tectons' functional amide moieties. However, two different morphologies of single molecules discriminating in-chain from end-chain positions are encountered. In addition, dimer structures are present, which appear as a central spot of changing brightness surrounded by four lobes. The peculiar shapes observed for 1 strongly indicate the existence of metastable flat-lying conformers, in particular related to the resolution of $\sigma$-bond $\mathrm{C}-\mathrm{C}$ (Scheme 1 ) and $\mathrm{C}-\mathrm{N}$ conformers owing to the symmetry-breaking effect of the surface.

Finally, upon adsorption at $300 \mathrm{~K}$ (Figure 1c) we observe regular, self-assembled linear chains consisting of several tens of tectons (each appearing as a bright dog-bone-like feature). ${ }^{[11]}$

The chains avoid hexagonal close-packed (hcp) stacking regions and dislocation lines, which means that their average length amounts to approximately 14 molecules or $9 \mathrm{~nm}$. The high degree of order within the chains indicates that they represent an equilibrated state of the system. The higher adsorption temperature thus yields a unique adsorption geometry for a largely dominating portion of the molecules. Consistently, further STM data recorded for coverages up to monolayer saturation reveal that the chain conformer is by far the most common species present in equilibrated systems and indicate the absence of formation of 2D molecular networks (see the Supporting Information).

In order to better characterize the conformation of 1) the isolated single molecules and 2) the in-chain molecules, NEXAFS measurements were carried out. With this technique, the orientation of functional moieties with respect to the substrate plane can be assessed. ${ }^{[12]}$ For the case (1), the molecules were deposited at $160 \mathrm{~K}$. At this temperature, mainly isolated molecules are present at the surface (see also Figure $1 \mathrm{a}$ ). For the analysis of (2), the samples were annealed for $5 \mathrm{~min}$ at $300 \mathrm{~K}$ to convert the molecules into the in-chain configuration (see also Figure 1c).

The four series of spectra (Figure $2 \mathrm{a}$ ) exhibit a pronounced dichroism, indicating well-defined molecular adsorption geometries. The fact that the resonances are sharp denotes a weak interaction of the molecules with the Au substrate. ${ }^{[13]} \mathrm{A}$ comparison of the grazing incidence carbon spectra (dashed lines, left column) highlights a remarkable change: three wellseparated peaks $(T=160 \mathrm{~K})$ develop into two peaks upon annealing to $300 \mathrm{~K}$, with the lower one featuring a shoulder. This repositioning of resonances indicates a change of chemical properties connected with the 1D self-assembly. The first $\pi^{*}$ resonance (binding energy $E_{\mathrm{B}}=285 \mathrm{eV}$ ) of the $C 1 \mathrm{~s}$ spectra can be attributed to the phenyl-ring $\pi$ system, while the $01 \mathrm{~s}$ edge shows a $\pi^{*}$ resonance $\left(E_{\mathrm{B}}=531.2 \mathrm{eV}\right)$ that originates from the two carbonyl double bonds in the molecular backbone. If $\theta$ is defined as the angle between the surface normal and the $E$ vector of the photon beam, an ideally flat-lying phenyl ring should result in a vanishing peak area for $\theta=90^{\circ}$ for the corre- 

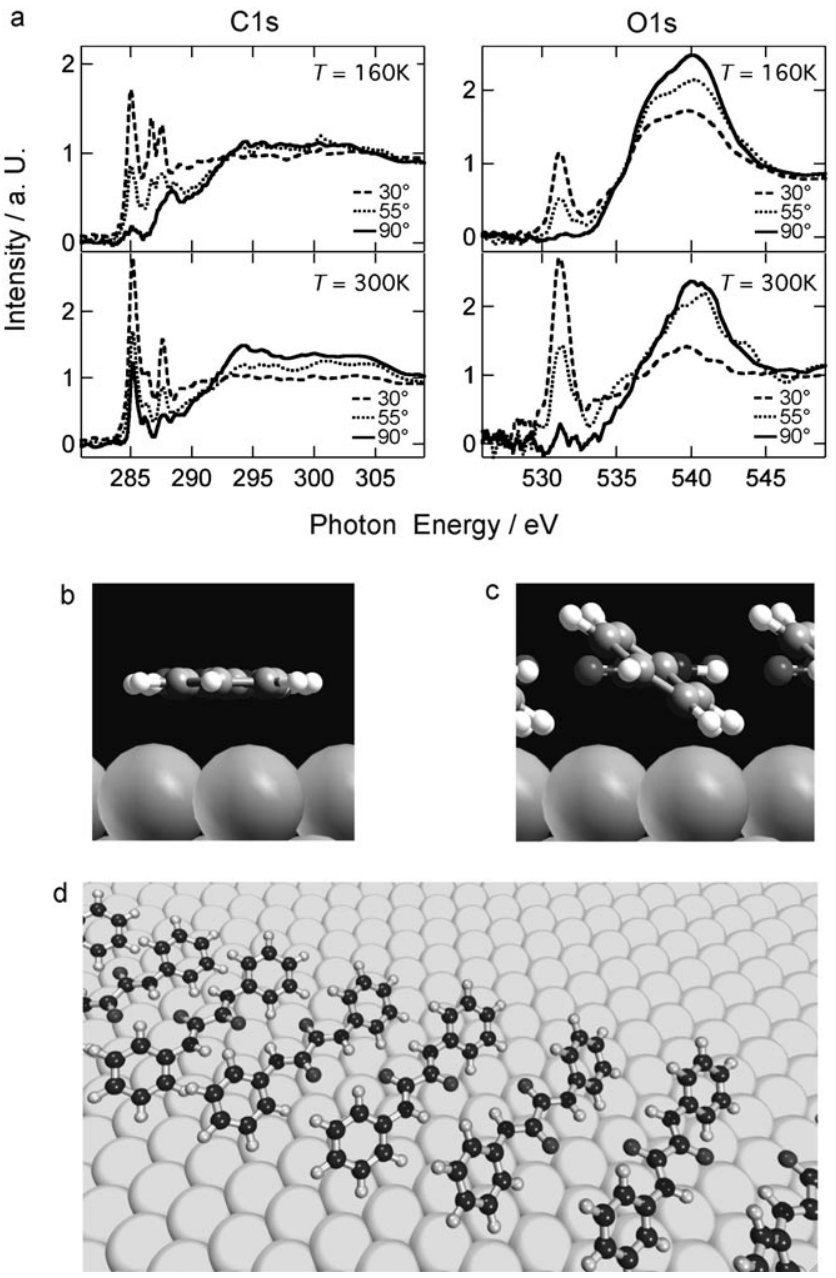

Figure 2. a) NEXAFS spectra of molecular adlayers deposited at $T=160 \mathrm{~K}$ (yielding single molecules, upper panels) and spectra of molecules annealed at $T=300 \mathrm{~K}$ (to give chain structures, lower panels). C $1 \mathrm{~s}$ edge (left column): The dichroism of the phenyl $\pi^{*}$ resonance at $285 \mathrm{eV}$ indicates essentially flat-lying phenyl rings for single molecules (upper panel) and a tilt of $\approx 35$ for molecules assembled in chains (lower panel). O 1s edge (right column): The dichroism of the $\mathrm{CO} \pi^{*}$ resonance at $531 \mathrm{eV}$ indicates a flat-lying inner group for single molecules (top) as well as for molecules assembled in chains (bottom). b) and c) Model representations of molecular conformations as indicated by NEXAFS for the low (b) and high (c) temperature cases. d) $3 D$ visualization of the chain structure as concluded from combined STM and NEXAFS results.

sponding signal. At low deposition temperatures, the phenylring signal is strongly reduced when going from $\theta=30^{\circ}$ (dashed curve) to $\theta=90^{\circ}$ (solid line), thus indicating a phenyl orientation nearly parallel to the substrate surface (Figure $2 \mathrm{~b}){ }^{[14]}$ In contrast, after annealing the chain structure, the spectra feature a remarkable intensity for $\theta=90^{\circ}$. Now the angular dependence of the peak intensity corresponds to a rotation of the phenyl groups of about $35^{\circ}$ with respect to the basal plane of the surface (Figure $2 \mathrm{c}$ ). Because in both cases the $01 \mathrm{~s}$ edge indicates a flat-lying inner part of the molecule 1 , the change of the phenyl orientation must originate from a conformational adaptation. A simple rotation of the entire molecule can be excluded. Further data for samples with higher molecular coverage $(0.3,0.5$, and 1 monolayer) show approxi- mately the same tilt, thus ruling out a coverage-dependent compression of molecules due to increasing lateral "pressure". Combining the data from STM and NEXAFS, we put forth a tentative model for the hydrogen-bonded 1D nanochains as depicted in Figure $3 \mathrm{~d}$.
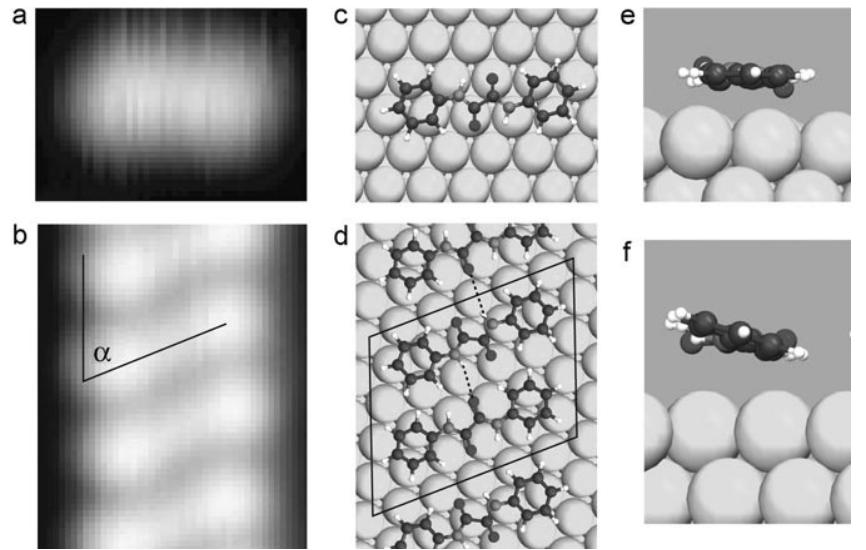

Figure 3. a) and b) STM images; c) and d) top views of energy-optimized conformations obtained from DFT calculations; e) and f) side views of energy-optimized conformations. Top [(a), (c), and (e)]: single molecule of $\mathbf{1}$; (c) has the same scale as (a) and (b). For the isolated molecule, the calculations predict phenyl rings lying flat on the surface, as shown in (e). Bottom [(b), (d), and (f)]: molecules of 1 inside a chain structure. DFT calculations (d) indicate that the chains are bound by one hydrogen bond per molecule (dotted lines between $\mathrm{O}$ atoms and $\mathrm{NH}$ groups). In ( $\mathrm{f}$ ), the side view of (d) shows that the phenyl rings of the relaxed structure are tilted by about $10^{\circ}$ with respect to the surface plane.

To rationalize the conformational adaptation of $\mathbf{1}$ in the supramolecular chain-forming process, DFT calculations were performed. In Figure 3, STM topography images (Figures 3 a and $b$ ) are compared with the top (Figures $3 c$ and $d$ ) and the side view (Figures $3 e$ and $f$ ) of the structurally relaxed models. The elongated STM appearance of a single molecule of 1 compares well with the straight conformation of an isolated molecule found in the calculation. Within the chains (Figure $3 \mathrm{~b}$ ), the molecules appear with two brighter lobes at the ends and a waist in the center. The bright lobes originate from the electron-rich $\pi$ systems of the phenyl rings. The single molecule appears as a more ellipsoidal shape. This difference is attributed to different imaging conditions, mainly a stronger convolution owing to the state of the tip. Concerning the 1D assemblies, the STM images of the in-chain molecules exhibit the same characteristic angle $\alpha$ between the axis of the molecule and the chain direction as that found in DFT.

Furthermore, the DFT results indicate that 1) the lowestenergy conformation of isolated molecules is s-trans (Figure $3 \mathrm{c}$ ) and 2) only one $\mathrm{N}-\mathrm{H} \cdots \mathrm{O}=\mathrm{C}$ hydrogen bond (Figure $3 \mathrm{~d}$, dotted lines) between adjacent molecules is formed within the chains. Two different conformers linked to each other define the unit cell (Figure $3 d$, thin line) of the chain structure, which represents an alternately ordered mixture. The alternation and the tilt angle between the main molecular axes and the chain axis imply that each conformer can only form hydrogen bonds with another molecule through either its $\mathrm{CO}$ or its $\mathrm{NH}$ groups, 
since in each molecule only one of these two groups will face the other group of adjacent molecules.

The two-molecule periodicity of this structure is reflected in two different, stretched hydrogen bond lengths (from $\mathrm{O}$ to $\mathrm{H}$ ) of 3.3 and $3.9 \AA$ in the structure, consistent with the intermolecular distances observed by placing scaled models over the STM images. The calculated bonding energy is $0.15 \mathrm{eV}$ per molecule in the optimized geometry. Furthermore, the calculations agree qualitatively with the NEXAFS results in predicting a flat geometry for the isolated molecule (Figure $3 \mathrm{e}$ ) and tilted phenyl rings for conformers within the chain structure (Figure $3 \mathrm{f}$ ). The calculated tilt angle of $10^{\circ}$ is, however, smaller than the experimental value of about $35^{\circ}$. This discrepancy may be due to the fact that the DFT code used here overestimates the $\pi$ system-metal interactions and thus introduces a spurious bias towards flatter adsorption geometries.

Both experimental and computational results indicate that isolated molecules of 1 lie flat on the $A u(111)$ surface with phenyl rings and amide moieties parallel to the substrate. Increasing the substrate temperature results in the self-assembly of $1 D$ chainlike structures in the fcc domains of the reconstructed $\mathrm{Au}(111)$ surface. Chain formation is driven by intermolecular hydrogen bonding. These bonds would be sterically hindered if the molecules stayed in a planar conformation. Upon rotation of the phenyl rings, the functional groups of adjacent molecules approach each other and form stronger bonds. Recent work on rubrene ${ }^{[15]}$ also showed that on the $\mathrm{Au}$ surface the molecule exhibited a different conformation than in the bulk. There, molecule-substrate interactions are the origin of the adaptation. In contrast, in our case the moleculemolecule interactions override the molecule-surface interactions and thus are able to drive the phenyl rotation. Furthermore, a resonant photoemission study of 4-fluorobenzenethiol on $\mathrm{Au}(111)$ recently demonstrated a strong dependence of the molecules' charge transfer properties on the angle between the substrate and the phenyl ring. ${ }^{[16]}$ Changing the angle by approximately $30^{\circ}$, which is comparable to the adaptation that we see, causes a transition from negligible charge transfer to fast charge transfer into the substrate.

In conclusion, our findings reveal that intermolecular noncovalent bonding between amide groups of adsorbed species with intramolecular degrees of freedom can override the competitive tecton-substrate coupling and lead to conformational adaptation in supramolecular organization. Similar mechanisms may drive the assembly of a variety of other systems. We suggest that molecular building blocks based on conformationally adaptive design can upgrade the tools of 2D supramolecular engineering and thus help to enhance the control over functional low-dimensional nanosystems.

\section{Experimental and Theoretical Section}

Compound 1 was synthesized according to literature protocols. ${ }^{[17]}$ The STM experiments were performed in an UHV system (base pressure of a few $10^{-11} \mathrm{mbar}$ ) equipped with standard sample preparation facilities and a home-built low-temperature scanning tunneling microscope. ${ }^{[18]}$ Images were taken in the constant cur- rent mode using a $\mathrm{W}$ tip. The tunneling bias $V_{t}$ is applied to the crystal with respect to the tip being grounded through the IV converter. The surface was prepared by ion-gun sputtering [ $p$ (Argon) $\left.\approx 3 \times 10^{-6} \mathrm{mbar}\right]$ at room temperature (RT) and annealing to $620^{\circ} \mathrm{C}$. The molecules have been deposited from a quartz crucible in a Knudsen-type cell held at $135^{\circ} \mathrm{C}$ onto the metal surface at temperatures as indicated. After deposition, the sample was cooled down to $10 \mathrm{~K}$ for STM. One monolayer (ML) is defined as an adlayer fully covering the surface.

NEXAFS measurements were performed at the BESSY II HE-SGM beamline. All NEXAFS spectra were taken with an energy resolution of $0.5 \mathrm{eV}$ at a substrate temperature of $160 \mathrm{~K}$. The NEXAFS raw data were treated to concentrate on the adlayer-related information; the details on data processing are described elsewhere. ${ }^{[19]}$ As a trade-off between a high percentage of statistically separated molecules and a large enough signal, the $C 1 \mathrm{~s}$ region data was obtained for $0.25 \mathrm{ML}$ coverage, while a $0.6 \mathrm{ML}$ coverage was necessary for the analysis of the $\mathrm{O} 1 \mathrm{~s}$ edge to compensate for the smaller number of $\mathrm{O}$ atoms per molecule.

DFT calculations were carried out using the Car-Parrinello method within the Projector Augmented Wave representation scheme. We used the Perdew-Wang 92 local density approximation for the exchange-correlation functional. ${ }^{[20]}$ The plane wave expansions were limited by a 28 Ry energy cut-off and the Brillouin zone sampling was limited to the gamma point only. A $0.25 \mathrm{eV}$ Fermi level smearing was used to deal with the metallic slabs as described elsewhere. ${ }^{[21]}$ All the calculations were performed in periodic boundary conditions. A vacuum region of $10 \AA$ was used to minimize the interaction between each gold slab and its periodically repeated images. The simulated gold slabs were composed of four atomic layers, with the atomic distances taken as calculated by first principles; no reconstruction was taken into account. The last layer was kept fixed during the damped dynamics simulations used to optimize the geometries.

\section{Acknowledgements}

This work was partially supported by the European Science foundation EUROCORES-SONS-FunSmarts network and the EC Sixth Framework Programme BIOMACH.

Keywords: density functional calculations - hydrogen bonds self-assembly $\cdot$ scanning probe microscopy $\cdot$ supramolecular chemistry

[1] J. M. Lehn, Proc. Natl. Acad. Sci. USA 2002, 99, 4763.

[2] L. J. Prins, D. N. Reinhoudt, P. Timmerman, Angew. Chem. 2001, 113, 2446; Angew. Chem. Int. Ed. 2001, 40, 2382; G. R. Desiraju, Acc. Chem. Res. 2002, 35, 565; T. Steiner, Angew. Chem. 2002, 114, 50; Angew. Chem. Int. Ed. 2002, 41, 48.

[3] S. De Feyter, F. C. De Schryver, Chem. Soc. Rev. 2003, 32, 139; J. V. Barth, G. Costantini, K. Kern, Nature 2005, 437, 671; J. V. Barth, Annu. Rev. Phys. Chem. 2007, 58, 375.

[4] J. V. Barth, J. Weckesser, C. Cai, P. Günter, L. Bürgi, O. Jeandupeux, K. Kern, Angew. Chem. 2000, 112, 1285; Angew. Chem. Int. Ed. 2000, 39, 1230; A. Dmitriev, N. Lin, J. Weckesser, J. V. Barth, K. Kern, J. Phys. Chem. B 2002, 106, 6907; S. Griessl, M. Lackinger, M. Edelwirth, M. Hietschold, W. M. Heckl, Single Mol. 2002, 3, 25; J. A. Theobald, N. S. Oxtoby, M. A. Phillips, N. R. Champness, P. H. Beton, Nature 2003, 424, 1029; S. Clair, S. Pons, A. P. Seitsonen, H. Brune, K. Kern, J. V. Barth, J. Phys. Chem. B 2004, 108, 19392; S. Stepanow, N. Lin, F. Vidal, A. Landa, M. Ruben, J. V. Barth, K. Kern, Nano Lett. 2005, 5, 901; R. Otero, M. Schöck, L. M. Molina, 
E. Laegsgaard, I. Stensgaard, B. Hammer, F. Besenbacher, Angew. Chem 2005, 117, 2310; Angew. Chem. Int. Ed. 2005, 44, 2270; M. Stöhr, M. Wahl, C. H. Galka, T. Riehm, T. A. Jung, L. H. Gade, Angew. Chem. 2005 117, 7560; Angew. Chem. Int. Ed. 2005, 44, 7394; M. E. Cañas-Ventura, W. Xiao, D. Wasserfallen, K. Müllen, H. Brune, J. V. Barth, R. Fasel, Angew. Chem. 2007, 119, 1846; Angew. Chem. Int. Ed. 2007, 46, 1814.

[5] T. Yokoyama, T. Kamikado, S. Yokoyama, S. Mashiko, J. Chem. Phys. 2004 121, 11993; M.-C. Blüm, E. Cavar, M. Pivetta, F. Patthey, W.-D. Schneider, Angew. Chem. 2005, 117, 5468; Angew. Chem. Int. Ed. 2005, 44, 5334 M.-C. Blüm, M. Pivetta, F. Patthey, W.-D. Schneider, Phys. Rev. B 2006, 73, 195409.

[6] F. Moresco, G. Meyer, K. H. Rieder, H. Tang, A. Gourdon, C. Joachim, Phys. Rev. Lett. 2001, 86, 672; X. H. Qiu, G. V. Nazin, W. Ho, Phys. Rev. Lett. 2004, 93, 196806; B. Y. Choi, S. J. Kahng, S. Kim, H. Kim, H. W. Kim Y. J. Song, J. Ihm, Y. Kuk, Phys. Rev. Lett. 2006, 96, 156106; J. Henzl, M. Mehlhorn, H. Gawronski, K. H. Rieder, K. Morgenstern, Angew. Chem. 2006, 118, 617; Angew. Chem. Int. Ed. 2006, 45, 603.

[7] M. Lingenfelder, G. Tomba, G. Costantini, L. C. Ciacchi, A. D. Vita, K. Kern, Angew. Chem. 2007, 119, 4576; Angew. Chem. Int. Ed. 2007, 46, 4492.

[8] M. Castonguay, J.-R. Roy, A. Rochefort, P. H. McBreen, J. Am. Chem. Soc 2000, 122, 518; S. Weigelt, C. Busse, L. Petersen, E. Rauls, B. Hammer K. V. Gothelf, F. Besenbacher, T. R. Linderoth, Nat. Mater. 2006, 5, 112.

[9] J. V. Barth, J. Weckesser, G. Trimarchi, M. Vladimirova, A. D. Vita, C. Cai, H. Brune, P. Günter, K. Kern, J. Am. Chem. Soc. 2002, 124, 7991; S. M. Barlow, R. Raval, Surf. Sci. Rep. 2003, 50, 201;K.-H. Ernst, Top. Curr. Chem. 2006, 265, 209.

[10] J. V. Barth, H. Brune, G. Ertl, R. J. Behm, Phys. Rev. B 1990, 42, 9307.

[11] The single dots at the chevron elbow sites are contaminations appearing after prolonged sample housing in the microscope.
[12] J. Stöhr, NEXAFS Spectroscopy, Springer, Heidelberg, 1991.

[13] C. Mainka, P. S. Bagus, A. Schertel, T. Strunskus, M. Grunze, C. Wöll, Surf. Sci. 1995, 341, L1055; G. Witte, K. Weiss, P. Jakob, J. Braun, K. L. Kostov C. Wöll, Phys. Rev. Lett. 1998, 80, 121.

[14] Note that isolated molecules flipping between two conformations with a tilt angle of $+30^{\circ}$ and $-30^{\circ}$ would not lead to an averaged NEXAFS signal indicating $0^{\circ}$. Thus, flipping isolated molecules are not conform with the NEXAFS data.

[15] D. Käfer, L. Ruppel, G. Witte, C. Wöll, Phys. Rev. Lett. 2005, 95, 166602.

[16] L. Wang, L. Liu, W. Chen, Y. Feng, A. T. S. Wee, J. Am. Chem. Soc. 2006, $128,8003$.

[17] R. Meyer, A. Seeliger, Ber. Dtsch. Chem. Ges. 1896, 29, 2640.

[18] S. Clair, Ph.D. thesis, available at http://library.epfl.ch/theses/thesis, Ecole Polytechnique Fédérale de Lausanne 2004; S. Clair, S. Pons, H. Brune, K. Kern, J. V. Barth, Angew. Chem. 2005, 117, 7460; Angew. Chem. Int. Ed. 2005, 44, 7294.

[19] M. E. Cañas-Ventura, F. Klappenberger, S. Clair, S. Pons, H. Brune, K. Kern, T. Strunskus, C. Wöll, R. Fasel, J. V. Barth, J. Chem. Phys. 2006, 125, 184710.

[20] P. E. Blöchl, C. J. Först, J. Schimpl, Bull. Mater. Sci. 2003, 26, 33; R. Car, M. Parrinello, Phys. Rev. Lett. 1985, 55, 2471; J. Perdew, Y. Wang, Phys. Rev. B 1992, 45, 13244.

[21] M. Stengel, A. De-Vita, Phys. Rev. B 2000, 62, 15283.

Received: May 25, 2007

Published online on July 18, 2007 